\title{
Evaluation of vacuum-assisted closure in the treatment of poststernotomy mediastinitis
}

Patrick W. Domkowski, MD, PhD

Monica L. Smith, BS

Denis L. Gonyon, Jr, MD

Carol Drye, $\mathrm{RN}^{\mathrm{b}}$

Mary Kay Wooten, $\mathrm{RN}^{\mathrm{c}}$

L. Scott Levin, MD ${ }^{\mathrm{b}}$

Walter G. Wolfe, MD ${ }^{\mathrm{a}, \mathrm{c}}$
From the Divisions of Cardiovascular and Thoracic Surgery $^{\mathrm{a}}$ and Plastic Surgery, Department of Surgery, Duke University Medical Center, Durham, NC, and The Durham Veterans Hospital, Department of Surgery, ${ }^{\mathrm{c}}$ Durham, NC.

Received for publication July 19, 2002; revisions requested Aug 2, 2002; revisions received Oct 10, 2002; accepted for publication Nov 8, 2002.

Address for reprints: Walter G. Wolfe, MD, Duke University Medical Center, Box 3507, Durham, NC 27710 (E-mail: wolfe001@mc.duke.edu).

J Thorac Cardiovasc Surg 2003;126:386-90

Copyright $\odot 2003$ by The American Association for Thoracic Surgery

$0022-5223 / 2003 \$ 30.00+0$

doi: $10.1016 / \mathrm{S} 0022-5223(03) 00352-0$
Objective: Poststernotomy mediastinitis, although infrequent, is a potentially lifethreatening complication of cardiac surgery that continues to have a significant morbidity and mortality despite aggressive therapy. Vacuum-assisted closure uses controlled suction to provide evacuation of wound fluid, decrease bacterial colonization, stimulate granulation tissue, and reduce the need for dressing changes.

Methods: One hundred two patients from Duke University Hospital, The Durham Veterans Administration Hospital, and referring institutions underwent vacuumassisted closure treatment. There were 63 men and 39 women, with a mean age of 67. The infection was noticed between postoperative days 8 and 34 , at which time the wounds were opened and debrided.

Results: Ninety-six of the 102 patients received vacuum-assisted therapy while the remaining 6 underwent daily multiple dressing changes without vacuum-assisted therapy. Fifty-three of the 96 patients required only sternal debridement, followed by wound vacuum therapy and closure by secondary intention, while the remaining 43 had an additional procedure. Of these, 33 patients underwent omental transposition and 10 patients had a pectoralis flap. The length of stay for all patients was $27 \pm 12$ days. This was related in part to intravenous antibiotics. Hospital mortality for all patients was $3.7 \%$ (4 patients). Two of these patients underwent vascular flap and succumbed to multisystemic organ failure, while the other 2 received only wound vacuum therapy following debridement and succumbed to overwhelming sepsis.

Conclusion: Vacuum-assisted drainage is an effective therapy for mediastinitis following debribement or before placement of a vascularized tissue flap.

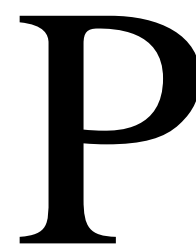

oststernotomy mediastinitis is an infrequent but potentially devastating complication after cardiac surgery. Reported incidences vary between $0.5 \%$ and $10 \%$, with an attendant mortality between $10 \%$ and $20 \% .^{1}$ This disparity is dependent on preoperative predictors, which include obesity, congestive heart failure, previous heart surgery, and duration of cardiopulmonary bypass. ${ }^{2}$ Treatment algorithms have evolved in an effort to reduce the mortality and the morbidity associated with mediastinitis. These include early aggressive debridement followed by either a pectoralis flap or an omental transposition.

The vacuum-assisted closure (VAC) technique evolved from a desire to develop a treatment for chronic debilitating wounds and was first described by Morykwas and colleagues in $1997 .{ }^{3}$ This device is currently indicated for chronic wounds such as stage III and IV pressure ulcers, along with venous, arterial, and neuropathic 
ulcers. Only recently has its use been expanded to the treatment of poststernotomy mediastinitis. The VAC device applies controlled suction to the wound, thereby increasing local blood perfusion, accelerating the rate of granulation tissue formation, decreasing tissue bacterial levels, and reducing the need for dressing changes. ${ }^{4}$ Studies have documented the effectiveness of the VAC device in the treatment of patients with poststernotomy mediastinitis, but these results have been limited to either case reports or small studies. ${ }^{5-7}$

In the present study, the effectiveness of the VAC system was evaluated as either an adjunct to conventional therapy (debridement and vascular flap) or as sole therapy (debridement plus VAC placement) for patients with post-cardiac surgery mediastinitis.

\section{Patients and Methods}

Between 1997 and 2002, 102 patients from Duke University Hospital, The Durham Veteran Administration Hospital, and referring institutions (both in and out of state) underwent treatment of poststernotomy mediastinitis. Data obtained from medical records included demographic information (age, sex, race, weight), time interval to mediastinitis presentation, prior operative procedures, wound VAC application, hospitalization stay, mediastinal intervention, and mortality. Culture results of infected median sternotomies were also reviewed. Length of stay was calculated after the onset of mediastinitis if it occurred during the same hospitalization as the initial cardiac surgery or the hospitalization from a separate mediastinitis admission. The Duke Institutional Review Board approved the data collection methods.

At the time of presentation, the sternal wound was debrided of foreign material and necrotic tissue and the wound vacuum device was applied. Patients with superficial wound infections or fat necrosis were not included in this study. The decision to use a vascular flap was not made until after a 5- to 7-day interval of wound vacuum therapy. Only patients who had a deep sternal infection requiring removal of all wires had to undergo an additional vascular flap procedure. The decision to proceed to either an omental flap or a pectoralis flap was made based on surgeon's preference.

\section{Results}

One hundred two patients underwent treatment of poststernotomy mediastinitis. There were 63 men and 39 women, with an average age of 67 years. Mediastinal infection was detected between 8 and 34 days after the initial cardiac procedure. Hospitalization resulting from mediastinitis was $27 \pm 12$ days (Table 1 ). Treatment of the 102 patients with poststernotomy mediastinitis (Figure 1) consisted of debridement, with a combination of dressing changes and/or VAC application (specifically, reconstructive procedures with either omental transposition or a pectoralis flap). Six patients did not receive wound vacuum therapy; instead they were treated with multiple wet to dry dressing changes. These 6 patients were seen early during the examined time
TABLE 1. Patient characteristics

\begin{tabular}{lc}
\hline Mean age (y) & 67 \\
Sex & \\
$\quad$ Male & 63 \\
$\quad$ Female & 39 \\
Infection detected (postoperative days) & $8-34$ \\
Length of hospitalization stay (days) & $27 \pm 12$ \\
Mortality & $3.7 \%$ \\
\hline
\end{tabular}

interval, when the use of the vacuum device was still limited. Of the 96 patients who did receive wound vacuum therapy, 43 had additional procedures. Of these patients, 33 received omental transposition and 10 received a pectoralis flap, while the remaining 53 required only wound debridement and were allowed secondary closure with a vacuumassisted therapy device already in place.

There were 4 deaths $(3.7 \%)$. Two of these patients underwent vascular flap procedures (1 omental transposition and 1 pectoral flap) and death was attributed to multisystemic organ failure. The other 2 received only wound vacuum therapy after debridement and succumbed to overwhelming sepsis, related in part to delayed referrals to our institution.

\section{Discussion}

Poststernotomy mediastinitis, albeit infrequent, is a dreaded complication. Numerous studies have reported an increase in morbidity and mortality, as well as a decreased long-term survival, after an initial successful treatment. In addition, it is financially costly to the health care system because of increased hospitalization, additional procedures, and incumbent nursing care. The treatment of poststernotomy mediastinitis has evolved since 1969, when Payne and Larson ${ }^{8}$ described the technique of wound debridement, primary sternal closure, and closed mediastinal catheter irrigation. This was followed in the early 1980s by the use of muscle flaps to fill dead space in the mediastinum and to cover the traumatized sternum. ${ }^{1}$ Myocutaneous flaps have allowed early closure and healing of sternal wounds; however, the mortality rate associated with mediastinitis remains between $10 \%$ and $20 \%{ }^{1}$ VAC emerged in 1997 as first described by Morykwas and Argenta ${ }^{3}$ and can be regarded as a method that combines the benefits of both closed and open wound treatment. Animal studies performed by Morykwas and colleagues showed that the application of subatmospheric pressure increased blood flow, increased rates of granulation tissue formation, and decreased tissue bacterial counts. ${ }^{3}$ Specifically, laser Doppler flow increased by 4 times compared with baseline, while tissue bacterial counts decreased by $21 \%$ versus control wounds treated at ambient pressure.

The VAC technique has many advantages including the stimulation of granulation tissue that may make secondary closure with a myocutaneous flap unnecessary. It also clears 


\section{Treatment of Posternotomy Mediastinitis}

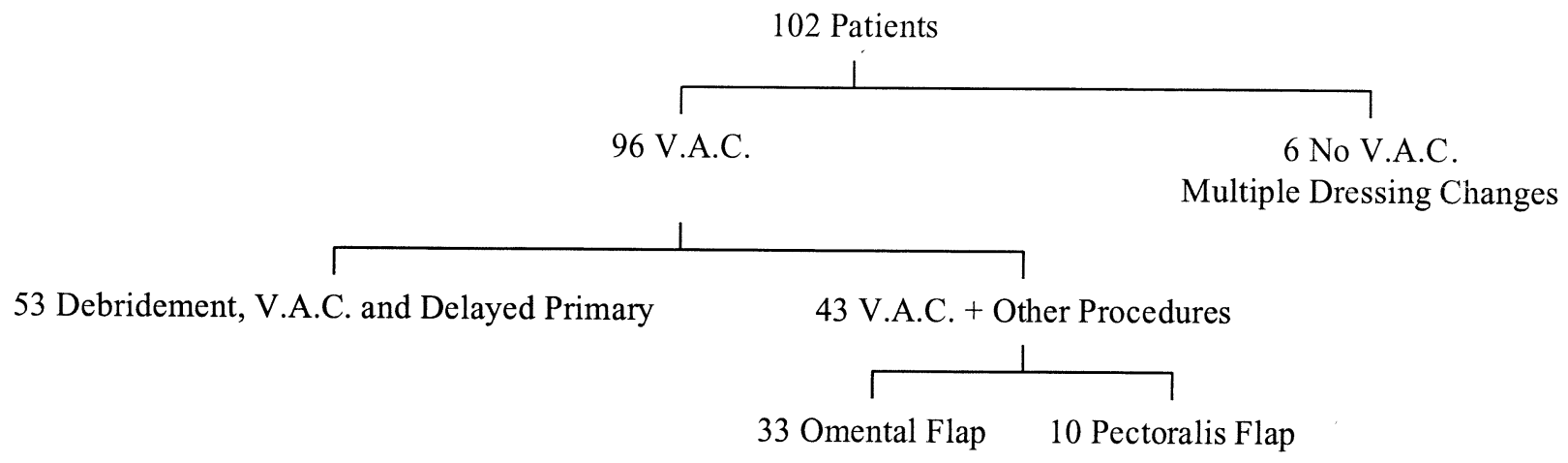

Figure 1. Surgical and VAC treatment of poststernotomy mediastinitis. VAC, Vacuum-assisted closure.

away secretions and toxic products by continuous suction, thus preventing fluid retention in the depth of the wound. This is accomplished with the creation of a mild negative atmospheric environment $(-125 \mathrm{~mm} \mathrm{Hg})$. Currently the use of intermittent suction is also being investigated. In addition, the polyurethane foam used in vacuum therapy only requires changing every 2 days, unlike the twice-daily changes needed in traditional wound dressings. Some patients are also able to go home with the wound vacuum in place by using a battery-operated portable suction device.

The clinical application of vacuum treatment for poststernotomy mediastinitis has been studied in 3 small series. Mendez-Eastman ${ }^{4,6}$ documented a case study of a 55-yearold woman who was readmitted for treatment of an infected sternal wound 11 days after coronary artery bypass grafting. The management of this patient included surgical incision and drainage of the sternum, sternectomy, and pectoralis and rectus abdominus muscle flaps. After the wound reopened, this patient then received hyperbaric oxygen treatment and VAC was applied to sternum. These additional techniques served as important complementary treatments essential to the healing process. Unlike the patient in this case report, the majority of the patients in our study underwent debridement and wound VAC therapy before a vascular flap procedure. Obdeijn and colleagues ${ }^{7}$ conducted a slightly larger study of 3 patients with poststernotomy mediastinitis who were treated with VAC. This study showed that the VAC applied to all patients made it possible to avoid the need for surgical closure with a vascularized muscle flap. In addition, no complications in wound healing were seen at a mean follow-up of 20 months. A study of 15 patients with poststernotomy mediastinitis treated with wound vacuum therapy was conducted by Tang and colleagues, ${ }^{9}$ who reported patients with varying severity of sternotomy wound infection. They reported complete wound healing in all survivors, including 3 who had sternal dehiscence infected by methicillin-resistant Staphylococcus aureus (MRSA). In addition, skin grafting was needed in 2 patients who had sternal dehiscence with mediastinitis. Berg and colleagues ${ }^{10}$ performed a retrospective analysis of patients with mediastinitis, comparing treatment with either a wound vacuum system or continuous irrigation. They found that the closed drainage system using a wound vacuum resulted in fewer treatment failures and a shorter stay in the hospital.

In a retrospective review of 72 patients with mediastinitis, Kirsh and colleagues ${ }^{11}$ used a closed drainage system with multiperforated rigid tubes (Redon drainage system) to evacuate the mediastinum. A mortality rate of approximately $35 \%$ was observed. This high number was in part attributed to the presence of MRSA in the culture medium. This type of closed drainage system varies significantly from the methods used in our study. The methods used by these authors created a strong negative pressure within the mediastinum $(-700 \mathrm{~mm} \mathrm{Hg})$. The pressure typically treated with wound VAC systems is 50 to $100 \mathrm{~mm} \mathrm{Hg}$. It is unclear whether this degree of negative pressure was detrimental to the patients. A recently study by Gustafsson and colleagues ${ }^{12}$ reported the novel use of monitoring plasma Creactive protein levels in guiding the VAC system. They reported 16 patients who had deep sternal infections at no mortality at 3 months after surgery. ${ }^{12}$

In the current study, the wound vacuum was used in patients who had either superficial sternal wounds (but not superficial wound infection; ie, above the sternal fascia) that did not necessitate removal of the wires and also in those patients who had a full sternal infection requiring removal of the wires. Patients underwent an additional procedure of either an omental transposition or a pectoralis flap. Milano and colleagues ${ }^{13}$ reported that those patients receiving an omental flap had both a shorter hospital stay and fewer early complications. ${ }^{13}$ Although we did not evaluate the data to 
determine if this difference existed in our group of patients, of those patients requiring a flap the majority did receive an omental transposition.

The results of these studies, although limited by the small number of patients evaluated, illustrate that vacuum therapy is an important treatment tool for the thoracic surgeon faced with poststernotomy mediastinitis. Moreover, its use also decreases the need for additional surgical reconstruction with vascularized flaps. The results from our study of 102 patients support these findings. Vacuum therapy proved to be an important adjunct to the treatment of poststernotomy mediastinitis. The overall incidence of poststernotomy mediastinitis at our institution is between $1 \%$ and $1.5 \%$. The wound vacuum system can be used either in combination with a vascular flap or as a primary therapy following debridement, which in turn allows secondary healing to proceed. The depth of the sternal infection influences the role of the wound vacuum, as either sole therapy for more superficial wounds or as adjuvant therapy for deeper wounds. Among those patients receiving vacuum therapy, 43 required vascularized flaps; however, 53 required no additional procedures except for wound debridement and secondary closure with the VAC system. Although a controlled randomized study would be necessary to determine if the wound vacuum device significantly lowered mortality and morbidity compared with historical treatment modalities, the data from this study support the role of the wound vacuum not only as an effective treatment tool but also to significantly lower overall mortality.

In conclusion, VAC is a useful asset in the treatment of poststernotomy mediastinitis. It may be used effectively before primary closure or as a preparation for secondary closure with vascularized tissue.

\section{References}

1. Gummert JF, Barten MJ, Hans C, et al. Mediastinitis and cardiac surgery-an updated risk factor analysis in 10,373 consecutive patients. J Thorac Cardiovasc Surg. 2002;50:87-91.

2. Milano CA, Kesler K, Archibald N, et al. Mediastinitis after coronary artery bypass graft surgery. Circulation. 1995;92:2245-51.

3. Morykwas MJ, Argenta LC, Shelton B, et al. Vacuum-assisted closure: a new method for wound control and treatment: animal studies and basic foundation. Ann Plast Surg. 1997;38:553-62.

4. Mendez-Eastman S. Use of hyperbaric oxygen and negative pressure therapy in the multidisciplinary care of a patient with nonhealing wounds. J Wound Ostomy Continence Nurs. 1999;26:67-76.

5. Raudat CW, Pagel J, Woodhall D, et al. Early intervention and aggressive management of infected median sternotomy incision: a review of 2242 open-heart procedures. Am Surg. 1997;63:238-42.

6. Mendez-Eastman S. Negative pressure wound therapy. Plast Surg Nurs. 1998;18:27-9.

7. Obdeijn MC, de Lange MY, Lichtendahl DH, de Boer WJ. Vacuumassisted closure in the treatment of poststernotomy mediastinitis. Ann Thorac Surg. 1999;68:2358-60.

8. Payne WS, Larson RH. Acute mediastinitis. Surg Clin North Am. 1969;49:999-1009.

9. Tang A, Ohri SK, Haw M. Novel application of vacuum assisted closure technique to the treatment of sternotomy wound infection. Eur J Cardiothorac Surg. 2000;17:482-4.
10. Berg HF, Brands WG, Geldorp RV, et al. Comparison between closed drainage techniques for the treatment of postoperative mediastinitis. Ann Thorac Surg. 2000;70:924-9.

11. Kirsh M, Mekontso-Dessap A, Houel R, et al. Closed drainage using Redon catheters for poststernotomy mediastinitis: results and risk factors for adverse outcome. Ann Thorac Surg. 2001;71:1580-6.

12. Gustafsson R, Johnsson P, Algotsson L, et al. Vacuum-assisted closure therapy guided by c-reactive protein level in patients with deep sternal wound infection. J Thorac Cardiovasc Surg. 2002;123:895-900.

13. Milano CA, Georgiade G, Mulbaier LH, et al. Comparison of omental and pectoralis flaps for poststernotomy mediastinitis. Ann Thorac Surg. 1999;67:377-81.

\section{Discussion}

Dr James W. Long (Salt Lake City, Utah). Dr Wolfe, you and your colleagues have made some very important contributions with a novel therapy. First, this is clearly the largest experience with vacuum-assisted closure for mediastinitis, 96 patients, and before this 15 maximum. Second, your mortality outcomes in this series are quite impressive at $3.7 \%$. Third, $55 \%$ of your patients were able to get by without vascular flap reconstruction. Obviously there are some important things that have been learned here. There are some challenges that are left, one of which that is not entirely clear from this paper is what role vacuum-assisted closure may be contributing. Your study design is primarily observational, with no statistically useful control group. You have a second group of small sample size, 6 patients, but not really characterized very well. One has to consider the possibility that your improved outcomes may not be due entirely vacuum-assisted closure. Certainly before this therapy results have been progressively improving. Even in your own institution in 1996 there was a $29 \%$ incidence of mediastinitis and by 1999 it is reduced to $10.5 \%$ when you used pectoralis flaps, and down to $4.8 \%$ with omental flaps without this therapy, quite close to the mortality results you had with this therapy. In the absence of a formal control group, we are not given a lot of information, either, to help us judge this historically. The question I have for you is: Do you believe that vacuumassisted closure therapy has been responsible for your improved mortality?

Dr Domkowski. Thank you, Dr Long, for reviewing our paper and for your comments and question. You are right, it is an observational and a retrospective analysis of vacuum-assisted closure. The way to do it would be a prospective trial in which we would randomize patients either to traditional treatment followed by vascular flap therapy if it were appropriate versus vacuumassisted. However, as this has been introduced to our institution, and obviously there is some bias involved, Dr Wolfe and I were very impressed with their initial results of vacuum-assisted therapy. In a significant number of patients, it has reduced the need for a second intervention (ie, omental or pectoral flap). We do believe that the use of this device is in large part responsible for the reduction in mortality. I think it is important to remember that the additional benefit, while it does reduce mortality, also saved many patients from an additional procedure, because with debridement in the operating room, with the infection going down to the bone, sometimes the wires would need to be tightened or a few taken out and then the vacuum-assisted device put in place. It is really quite remarkable that the physiology behind this vacuum does promote stimulation of granulation tissue, at least subjectively, again, to be 